\title{
Hipertensión arterial y consumo de sal en pediatría
}

\author{
CARLOS SAIEH A. ${ }^{1}$, EDDA LAGOMARSINO F. ${ }^{2}$ \\ 1. Profesor Agregado de Pediatría, Universidad de Chile, Nefrólogo Departamento de Pediatría, Clínica Las Condes. \\ 2. Profesora Titular de Pediatría, Nefróloga Hospital Clínico Pontificia Universidad Católica.
}

\begin{abstract}
\section{Arterial hypertension and salt intake in pediatrics}

Pediatric arterial hypertension (AH) is an underdiagnosed disease, with a known prevalence of $2-3 \%$. Its preventive management should begin early and includes life-style changes and diet salt reduction to a

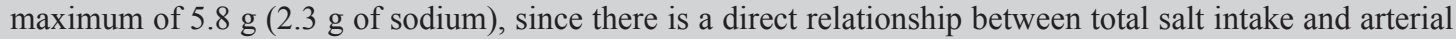
blood pressure. It has been previously shown that in populations with low salt diet (less than $3 \mathrm{~g}$ ), AH is rare and it does not increase with age. It has been estimated that $77 \%$ of salt found in regular diets comes from processed food. Mechanisms involved in salt intake and high blood pressure are analyzed in this paper. Arterial hypertension secondary to renal diseases and other pathologies are excluded. Considering renal physiology, the role of the kidney is crucial in arterial blood pressure regulation, through the capacity to affect the salt and water excretion; therefore, controlling total blood volume. The relationship between salt and $\mathrm{AH}$ in the newborn and older children, as well as genetic aspects of this disease, are discussed. In conclusion, there are biological and behavioural risk factors that can be modified in young population. It is necessary to promote these changes through active, as well as passive, prevention strategies. A government public health policy including educational publicity campaigns, permanent media information and accessible health food labelling is essential. Habits, old customs and trends need to be changed through a multifactorial approach to groups, families and community. The pediatrician should lead this effort.

(Key words: hypertension, blood pressure, risk factors, life-styles, salt intake).

Rev Chil Pediatr 2009; 80 (1): 11-20
\end{abstract}

\section{RESUMEN}

La hipertensión arterial (HA) en pediatría es una patología sub diagnosticada, con una prevalencia de alrededor de un 2 a 3\%. Su prevención debe comenzar precozmente con indicaciones de estilos de vida saludables, en especial reducción de sodio a $2,3 \mathrm{~g}$ equivalentes a 5,8 g de cloruro de sodio diarios, dada la relación directa entre cantidad de sal de la dieta y la presión arterial. En poblaciones humanas con dietas conteniendo menos de $3 \mathrm{~g}$ de sal por día la HA es infrecuente y no aumenta con la edad. La mayor parte del sodio ingerido se aporta por los alimentos elaborados industrialmente: el $77 \%$ se obtiene de los alimentos

Trabajo recibido el 28 de agosto de 2008, devuelto para corregir el 28 de octubre de 2008, segunda versión el 10 de diciembre de 2008, aceptado para publicación el 28 de diciembre de 2008.

Correspondencia a:

Dr. Carlos Saieh A.

E-mail: csaieh@clinicalascondes.cl 
procesados. En esta comunicación se revisan los mecanismos que relacionan las alzas tensionales con la ingesta de sal. Se excluyen los mecanismos que producen HA en las enfermedades renales y otras formas de HA secundarias. Desde el punto de vista fisiológico el riñón tiene un papel primordial en la regulación de la presión arterial, entre otros por su capacidad de modificar la excreción de agua y sal y con ello regular el volumen de sangre circulante. Se analiza la relación sodio/HA en el recién nacido y en el niño mayor y algunos aspectos genéticos de esta enfermedad. Se concluye que hay factores de riesgos biológicos y conductuales en los jóvenes que son modificables. Es necesario promover cambios en estos grupos a través de estrategias de prevención poblacional tanto activas como pasivas. Esto requiere un compromiso de políticas públicas que incluyan campañas educativas, manejo de la publicidad y fácil identificación de los alimentos saludables. Deben intervenirse los hábitos, costumbres y tendencias a través de acciones con un enfoque multifactorial, familiar, grupal y comunitario, siendo el pediatra quien debe liderar este desafío.

(Palabras clave: hipertensión, presión arterial, factores de riesgo, estilos de vida, consumo de sal).

Rev Chil Pediatr 2009; 80 (1): 11-20

\section{Introducción}

En la población adulta la hipertensión arterial (HA) es una patología frecuente, describiéndose en la mayoría de los países de Sudamérica una prevalencia que varía entre 8 y $30 \%$. Estas cifras son similares a las reportadas en el resto del mundo, lo que demuestra que el problema de la HA es común globalmente, afectando eso sí a algunas poblaciones más que a otras ${ }^{1}$. Por otro lado, la carga de enfermedad atribuible a la HA a nivel global es considerable, estimándose que causa 7,1 millones de muertes prematuras, siendo responsable del $4,5 \%$ de la carga de enfermedad en el mundo y del $45 \%$ de las patologías cardiovasculares ${ }^{2}$.

La enfermedad cardiovascular (CV) es la principal causa de muerte en Chile y es la tercera causa de invalidez $z^{3}$. Se considera que su prevalencia continuará en ascenso en el mundo ${ }^{4}$. Existen innumerables evidencias de la importancia de la HA como factor de riesgo $\mathrm{CV}$, especialmente sobre las enfermedades coronaria y cerebrovascular. Recientes investigaciones demuestran que una pequeña disminución en las cifras de PA logra reducciones significativas en las consecuencias asociadas a la HA. Así una baja de $2 \mathrm{~mm} \mathrm{Hg}$ de presión arterial diastólica resulta en una reducción de un $14 \%$ en el riesgo de ataque cerebral y de $6 \%$ en enfermedad coronaria ${ }^{5}$.

La PA en los niños sigue un patrón que se mantiene hasta la tercera o cuarta década de la vida, lo que sugiere que el valor de la presión en los primeros años de vida es un indicador de riesgo de ser hipertenso en la vida adulta, y que una correcta intervención en los estilos de vida en edades tempranas puede llevar a una reducción de la incidencia de hipertensión ${ }^{6,8}$.

En el contexto de estos hábitos saludables, la restricción de la ingesta de sodio ha sido ampliamente estudiada y su efecto, como veremos más adelante, sobre la PA ha sido fehacientemente reconocido como favorable en el manejo de la $\mathrm{HA}^{9-10}$, pero implementar estas restricciones requiere de políticas públicas claras que deben iniciarse precozmente en la edad pediátrica.

\section{Hipertensión arterial en pediatría}

La HA es una patología sub diagnosticada en los niños, con una prevalencia de alrededor de un 2 a $3 \%^{11-12}$. A la luz de los conocimientos actuales se puede asegurar que la presión elevada tiene sus inicios en la niñez y en algunos casos incluso en la vida intrauterina, con tendencia a la perpetuación de los niveles de PA en el tiempo, es decir, que aquellos sujetos con valores de presión altos, tendrán en la edad adulta mayor riesgo de ser hipertensos que los niños con valores en los percentiles bajo $90^{13}$.

Evaluaciones clínicas realizadas en pacientes hipertensos, en relación al tratamiento no farmacológico o estilos de vida saludables, han 
demostrado ser beneficiosos para lograr descensos en las cifras de PA. Además, al adquirir estos hábitos saludables, se disminuye el riesgo de enfermedad CV, accidente vascular encefálico, diabetes mellitus tipo $2 \mathrm{y}$ enfermedad renal crónica. Su prescripción y cumplimiento es el fundamento para un buen tratamiento antihipertensivo. Se propicia como tratamiento único en pacientes con pre HA o con HA grado 1 sin compromiso de órganos blancos, o agregado al tratamiento farmacológico en pre hipertensos con factores de riesgo cardiovascular e HA grado 1 y $2^{14,18}$.

En la edad pediátrica hay suficiente experiencia como para insistir en que la prevención debe comenzar desde los primeros meses de vida con indicaciones de alimentación saludable y control anual de peso, talla y PA, por lo menos desde los tres años de edad ${ }^{12}$. Los estilos de vida saludable que han demostrado ser eficaces en reducir los niveles de presión arterial son las siguientes:

- Actividad física aeróbica en forma regular, mínimo tres veces por semana.

- Mantención del peso corporal en rangos normales (índice de masa corporal entre $\left.18.5-24.9 \mathrm{~kg} / \mathrm{m}^{2}\right)$.

- Evitar o limitar, en los adolescentes, el consumo de alcohol a no más de $30 \mathrm{ml}$ y $15 \mathrm{ml}$ de etanol al día.

- Reducir el consumo de sodio en la dieta a no más de 2,3 g de sodio o 5,8 g de cloruro de sodio.

- Mantener una ingesta adecuada de potasio en la dieta.

- Consumir alimentación con bajo contenido en grasa total y especialmente en grasas saturadas.

\section{El recién nacido y el balance de sodio}

En el recién nacido $(\mathrm{RN})$ existe un balance negativo de sodio en los primeros días de vida, evento auto limitado asociado a la contracción del volumen extracelular. Los cambios en la concentración plasmática de sodio a esta edad reflejan principalmente cambios en el balance de agua más que en el de sodio. El balance de agua tiende a ser positivo en el primer día debido al aumento en la secreción de vasopresina asociada al parto y a la baja tasa de filtración glomerular. Después del nacimiento hay una caída en la resistencia vascular renal que produce un mayor flujo sanguíneo renal y una mayor perfusión a los nefrones corticales, resultando en mayor filtración glomerular, aumento en la diuresis y balance negativo de agua en los siguientes días de vida posnatal.

La influencia de factores hormonales relacionados con la homeostasis del agua y del sodio varía en el RN comparado con el adulto. Existe, por ejemplo, una respuesta renal disminuida a la vasopresina, que se traduce en una menor capacidad concentradora de la orina. Las concentraciones de renina y aldosterona plasmática son altas en los $\mathrm{RN}$ tanto prematuros como de término, sin embargo, la respuesta tubular a la acción aldosterónica es baja a menores edades gestacionales. Esto da cuenta de una mayor incapacidad de retener sodio $\mathrm{y}$ excretar K en el prematuro ${ }^{19}$.

Respecto al manejo renal del sodio, existen diferencias en la fracción excretada de sodio (FeNa) con el adulto, que excreta menos del $1 \%$ del sodio filtrado. En prematuros menores de 30 semanas de gestación, el FeNa puede exceder el 5\% durante los primeros 3 días de vida, mientras que en el RN de término alcanza alrededor del $2 \%$. Se ha postulado un desbalance funcional glomérulo tubular como el responsable de este alto $\mathrm{FeNa}$ previo al nacimiento, sin embargo, otras teorías sugieren que la reabsorción de sodio por el riñón fetal está relacionada íntimamente con la tasa de filtración glomerular.

Se cree que la mayor excreción basal de sodio en RN pre término comparada con los de término es secundaria a la capacidad disminuida del túbulo distal del prematuro de reabsorber el sodio adecuadamente. En el RN de término, inmediatamente después del parto (2-4 horas), se produce un aumento transitorio del $\mathrm{FeNa}$, debido a la redistribución del flujo sanguíneo intrarrenal, aumento en la liberación de sustancias natriuréticas, y a cambios en la secreción de diferentes hormonas involucradas en la homeostasis del sodio. Después de las 4 horas se aprecia una rápida disminución en la excreción de sodio, llegando entre 24-48 horas a un FeNa de alrededor del $1 \%{ }^{19}$.

La ingesta y la excreción renal de sodio en condiciones normales operan en un equilibrio 
que mantiene estable la PA, el líquido extracelular y el balance de sodio. En respuesta a una ingestión de sodio, el balance se restablece gracias a cambios en la tasa de filtración glomerular, de la presión oncótica e hidrostática en los capilares peri tubulares, en el flujo sanguíneo renal y su distribución, la reabsorción de sodio por las células tubulares renales y el nivel circulante de hormonas que influyen el balance de sodio y la resistencia al flujo sanguíneo renal.

El volumen intravascular depende fundamentalmente del sodio. La angiotensina II estimula la reabsorción de sodio y agua independientemente de su efecto sobre la producción de aldosterona. La aldosterona también estimula la reabsorción de sodio a nivel del túbulo contorneado distal; este aumento en la reabsorción de sodio lo incrementa en el plasma, lo que estimula la producción de ADH y a su vez aumenta la reabsorción de agua. Los factores que estimulan la producción de aldosterona son la angiotensina II, el aumento de los niveles de potasio sérico, la contracción del volumen vascular y la hormona adrenocorticotrófica. El efecto de esta última probablemente sea menos importante ${ }^{20}$.

\section{Factores genéticos y ambientales en la $\mathrm{HA}$}

Las determinantes genéticas juegan un rol importante en la PA pediátrica. La etiología de la HA está basada en un modelo multifactorial de genes y ambiente. Los genes pertenecen a hormonas, enzimas, receptores y proteínas ubicadas en diversos órganos como riñón, glándulas suprarrenales, corazón, cerebro, páncreas e hígado. Son genes de expresión dominante, recesivos y ligados al sexo ${ }^{21}$. Sobre estos genes operan los factores ambientales constituidos por el aumento del consumo de sodio y peso corporal, factores que, actuando independientemente o asociados, aumentan la PA en la población general y/o en individuos aislados. Existe una mayor correlación entre PA de la madre con su hijo que entre el padre y su hijo, sugiriendo también una influencia directa prenatal. Los mecanismos genéticos relacionados con la PA incluyen flujo eritrocitario de sodio, excreción de kalicreína en orina, fenotipo II de la haptoglobina, sensibilidad a la sal, niveles anormales de lípidos plasmáticos y de resisten- cia a la insulina. A futuro podrá identificarse aquellos niños con riesgo genético de HA, pero en la actualidad estas determinantes no pueden ser intervenidas ${ }^{22}$.

Desde el punto de vista de los factores ambientales existe una correlación inversa entre el peso de nacimiento y la presión arterial sistólica, la que es mayor cuando a la baja de peso al nacer se suma un aumento del peso placentario, ambos indicadores de desnutrición. Esta tendencia está asociada a resistencia a la insulina e hiperlipidemias y a la presencia de catecolaminas que estimularían la angiotensina II. La desnutrición materna se traduce entre otras cosas, en una menor expansión del volumen sanguíneo, que parece ser la alteración materna que más directamente repercute en el crecimiento fetal al limitar la expansión del débito cardíaco y secundariamente, la perfusión sanguínea del útero y la placenta. Las madres de niños con retardo de crecimiento intrauterino tienen menor volumen plasmático, menor débito cardíaco y mayor resistencia vascular periférica ${ }^{23}$.

La enfermedad hipertensiva se ha interpretado como una adaptación del feto frente a un medio intrauterino adverso. La desnutrición en períodos de rápido crecimiento, como en la vida intrauterina, conlleva cambios permanentes estructurales y fisiológicos en un amplio rango de órganos y tejidos. Este fenómeno se conoce como programación. El peso de nacimiento no está determinado genéticamente, Por esto, las investigaciones están centradas en los aspectos nutricionales y metabólicos, ya que en ellos está la clave que permitirá acciones preventivas de la enfermedad cardiovascular ${ }^{24}$.

Las fórmulas lácteas actualmente en uso, cumplen los requerimientos de la American Academy of Pediatrics in Nutrition (20-60 mg de Na por $100 \mathrm{Kcal}$ ). Este nivel de ingesta se triplica o cuadruplica si se administra una fórmula no regulada y se multiplica por 10 cuándo se introducen los sólidos. Los lactantes sanos son capaces de adaptarse muy bien a cambios moderados en la ingesta de sal, sin embargo, pese a que pueden compensar la restricción de la ingesta de sodio casi con la misma eficiencia que los adultos, tienen limitaciones en su capacidad para excretar sobrecargas ${ }^{25}$. 
El destete precoz produce modificaciones metabólicas, no sólo referidos al contenido de sodio y la hiperosmolaridad consecuente, sino que incrementa la obesidad al producir un aumento en la velocidad de síntesis de ácidos grasos y disminución de su oxidación, aumento del contenido adiposo, disminución de la neoglucogénesis y modificaciones de los niveles plasmáticos de algunas hormonas, con bruscos descensos del glucagón y aumento de la insulina.

Conociendo cuáles son los factores de riesgo de HA modificables en la niñez se pueden considerar estrategias para prevenir la HA en el adulto.

\section{Fisiopatología de la HA y el cloruro de sodio}

Revisaremos los mecanismos que relacionan las alzas tensionales con la ingestión de sodio, quedando excluidos aquellos que producen HA en las enfermedades renales, suprarrenales y otras formas de HA secundaria.

El riñón tiene un papel primordial en la regulación de la PA, no sólo por su capacidad de modificar la excreción de agua y sal y con ello regular el volumen de sangre circulante, sino también por su complejo comportamiento como órgano endocrino productor de renina, la que al pasar a la sangre transforma la angiotensina I en angiotensina II, potente agente vasoconstrictor que induce directamente la síntesis de endotelina I para potenciar el efecto vasoconstrictor. Las dos sustancias responsable de la disminución del oxido nítrico potenciando todavía más la acción vasoconstrictora. El riñón es uno de los órganos productores de calicreína que, al igual que la renina, al liberarse en el plasma actúa sobre el cininógeno, liberando la bradicinina o la colidina, de intensa acción hipotensora, contrabalanceando así la acción de la renina angiotensina ${ }^{26}$.

En las fases iniciales de la HA se encuentra un aumento del gasto cardíaco y del flujo sin cambios en la resistencia periférica. El aumento posterior de ésta correspondería a un mecanismo adaptativo para disminuir el gasto cardíaco. La mayor ingesta de sal se acompaña de vasoconstricción y el aumento de radicales oxidados aumenta las sustancias vasoconstrictoras endoteliales. Esta interpretación fisiopatológica se acerca a lo observado en individuos genéticamente predispuestos, donde deben confluir una serie de factores ambientales, como el consumo exagerado de sal, para desencadenar hipertensión ${ }^{27,28}$.

La aparición de HA está generalmente subordinada a la existencia de uno o más factores predisponentes, que son los mismos que contribuyen a una mayor morbimortalidad cardiovascular. Se resumen en sobrepeso, vida sedentaria y falta de ejercicio e hipercolesterolemia. Mención aparte merece la exagerada ingesta de sal.

\section{Hipertensión arterial y consumo de sal}

La raza humana está genéticamente programada para consumir menos de $1 \mathrm{~g}$ de sal al día, pero en la mayoría de los países la dieta contiene entre 6 y $12 \mathrm{~g}$ al día ${ }^{29}$. Aquellos que consumen grandes cantidades de sal tienen un aumento consistente de los valores de PA con la edad. Independientemente de la PA, el alto consumo de sal produce incremento de la masa ventricular izquierda y mayor incidencia de enfermedades $\mathrm{CV}^{30}$.

En Texas un grupo de chimpancés, animales genéticamente muy parecidos a los humanos, recibió inadvertidamente una dieta conteniendo entre 6 y $12 \mathrm{~g}$ de sal por día. La PA se comportó de la misma manera que en los humanos, incluso aumentando progresivamente con la edad ${ }^{31}$. Para corroborar este efecto se alimentó a un conjunto de chimpancés con su comida habitual, es decir frutas y verduras, conteniendo menos de $1 \mathrm{~g}$ de sal por día y a otro grupo similar, tomado como control, con dieta rica en sal por 20 meses, al cabo de los cuales la PA aumentó significativamente en el últimos, retornando a valores normales al restituirles su alimentación habitual baja en sodio ${ }^{32}$.

Existe una relación directa entre la cantidad de sal de la dieta y los valores de PA. Como ejemplo se cita lo observado en las Islas Marshall (Pacifico Sur), cuya población tiene un consumo diario de sal de $8 \mathrm{~g}$ y una prevalencia de $8 \%$ de HA comparándola con la población del Norte de Japón, con un consumo de $25 \mathrm{~g}$ de sal diario y una prevalencia de $38 \%$ de $\mathrm{HA}^{33}$. En poblaciones humanas con dietas conteniendo 
menos de $3 \mathrm{~g}$ de sal por día la HA es poco frecuente y no aumenta con la edad.

En 1963 Cruz-Coke realizó un estudio en Isla de Pascua en 179 individuos, de los cuales 129 permanecieron en la isla y 50 emigraron a Chile continental. Se comprobó un ascenso de la PA a lo largo de la edad y aparición de HA solamente en aquellos sujetos que se trasladaron al continente ${ }^{34}$.

Karppanen en Finlandia ${ }^{35}$ publica algunas evidencias relacionando el alto consumo de sal en la población con aumento de la PA mientras que su restricción contribuye a mejorar esta situación. En los últimos 30 años una disminución de alrededor de un tercio de la sal ingerida por los finlandeses produjo una caída de la presión sistólica y diastólica en más de $10 \mathrm{mmHg}$ en promedio, sin existir ninguna manifestación de efectos indeseables por la reducción del sodio. Un reciente meta-análisis de publicaciones relacionadas con reducción de sal en la dieta de los niños corroboró una significativa caída de PA al disminuir la ingesta de sodio ${ }^{36}$.

La alimentación ha tomado un lugar preponderante como medio para lograr una reducción de la PA. En estudios realizados en poblaciones con dietas especiales, como la mediterránea y la dieta DASH (Dietary Approaches to Stop Hypertension), se logra importantes descensos de la presión. Así, con una dieta hiposódica con $80 \mathrm{mEq}$ de sodio $(4,7 \mathrm{~g}$ de sal) se obtiene un descenso de 5,0 y $3,0 \mathrm{~mm} \mathrm{Hg}$ para las presiones sistólica y diastólica respectivamente. La importancia del sodio queda de manifiesto en el estudio DASH, en el cual la alimentación rica en frutas y verduras con un aporte de sodio de $65 \mathrm{mEq}(3,8 \mathrm{~g}$ de sal) produce una disminución adicional de la presión sistólica en $7 \mathrm{~mm} \mathrm{Hg}$, comparada con el mismo régimen sin restricción de sodio ${ }^{37,39}$.

Numerosos estudios han establecido que la mayor parte del sodio ingerido se aporta por los alimentos elaborados industrialmente. Mattes ${ }^{40}$, publica que el $77 \%$ del sodio se obtiene de los alimentos procesados y de los restaurantes, un $12 \%$ proviene de los alimentos naturales, un $6 \%$ se agrega en la mesa y un 5\% durante la preparación. Estas cifras no difieren mucho de lo observado en nuestro país, en donde se estima que el $75 \%$ del sodio presente en la dieta se añade a los alimentos durante su procesamiento o manufactura y sólo el $10 \%$ procede de los alimentos naturales.

El cloruro de sodio es la principal fuente del sodio en los alimentos. Sin embargo, se debe tener en cuenta que hay otras fuentes, como son el glutamato monosódico, contenido en la salsa de soya y también en otros aditivos de alimentos, como el benzoato de sodio, nitrato de sodio, pirofosfato de sodio. La sal es altamente utilizada en la industria de alimentos para intensificar el sabor, en su preservación y procesamiento. En consecuencia tenemos alimentos con alto contenido de sodio que pueden ser encontrados en todos los supermercados. Dentro de ellos mencionamos los más frecuentes en nuestro medio:

Fiambres o embutidos (salchichón, mortadela, chorizo, salame, salchichas, etc), carnes ahumadas, patés, pescados y mariscos en conserva o ahumados, pepinillos en vinagre, aceitunas, cebollitas, alcaparras, quesos, mantequilla, platos preparados industrialmente, alimentos precocidos, concentrados de caldo, sopas de sobre, salsas y aliños (mayonesas, mostaza, ketchup, etc), productos de cóctel (maní, papas fritas, ramitas), pan y galletas.

En nuestro país, en un estudio realizado con la Junta Nacional de Auxilio Escolar y Becas (JUNAEB), se encontró que un servicio de almuerzo tipo entrega $650 \mathrm{Kcal}$ aportando una ingesta de sal de 5,26 $\pm 1,54 \mathrm{~g}$. Si extrapolamos una dieta diaria de $1500 \mathrm{Kcal}$ a cada niño, les estamos aportando alrededor de $11 \mathrm{~g}$ de sal por día ${ }^{41}$.

En USA, 1999, en un taller relacionado con la ingesta de sodio e hipertensión arterial, se concluyó que: "Existen suficientes evidencias acerca de la relación entre la ingesta elevada de sodio y la HA y otras condiciones cardiovasculares. La población norteamericana come más sodio que el que fisiológicamente necesita. Una estrategia poblacional amplia para reducir la sal en la alimentación es importante para reducir la HA en la población"42. El Instituto de Medicina de la Academia Nacional de USA recomienda que la ingesta máxima de sodio para un adulto sano debe ser de $2300 \mathrm{mg}$ diarios. El gobierno de Canadá adopta esta recomendación y establece que para el año 2020 la ingesta de sodio 
en los adultos deberá estar entre 1200 y 2300 mg por día ${ }^{43}$.

A pesar de estar ampliamente acreditado el efecto de la sal sobre la PA, se asume que este efecto es reversible. Sin embargo, Tekol, basado en estudios realizados en animales de experimentación, a quienes se les produjo HA mediada por sal, demuestra que hay efectos reversibles e irreversibles. Estos últimos se manifiestan porque la PA no vuelve a los valores iniciales una vez suspendida la sal. La evidencia actual indica que este fenómeno se puede producir en etapas de la vida intrauterina, durante el amamantamiento y en el período prepuberal. No hay investigaciones en humanos disponibles al respecto, pero basados en esta hipótesis, si se quiere disminuir la $\mathrm{HA}$ en la población se debe reducir la ingesta de sal, especialmente en el embarazo, durante la lactancia y en la niñez ${ }^{44}$.

La excesiva ingesta de sal se ha tornado tan común, que Tekol ${ }^{45}$ se refiere a la "adicción" a la sal, dado que al inicio de su abstinencia aparece anorexia y a veces náuseas, se reconoce una gran dificultad para restringirla, existe una evidente falta de éxito en las campañas poblacionales para disminuir la ingesta de sodio y aún más, no se logra cumplimiento de los pacientes hipertensos en reducir su consumo, a pesar de conocer los efectos deletéreos del sodio en su organismo. Quizás si se reconociera a la sal como adictiva sería más fácil realizar campañas para la disminución de su ingesta diaria.

\section{Estrategias de prevención}

Se denominan estrategias de prevención poblacionales aquéllas que se centran en lograr una pequeña disminución de las cifras de PA en toda la población. Con ello se disminuyen más las tasas de enfermedades $\mathrm{CV}$ que si se trabaja sólo con el grupo de pacientes hipertensos. Pueden ser activas o pasivas. Ambos enfoques son complementarios y no deben presentarse como excluyentes.

Una demostración de la eficacia de una estrategia pasiva lo dio el proyecto Exeter Andover USA. Durante un período de dos años se compararon los resultados de una dieta pobre en sal, entregada en una escuela $(20 \%$ menos de sal) en relación a otra que no se intervino. El promedio de la PA no aumentó en los estudiantes con dieta baja en sodio y sí lo hizo en los no intervenidos. El análisis de multivarianza de las diferencias entre ambos grupos, mostró que los efectos estimados generales de la intervención dietética fueron $-1,7$ $\mathrm{mmHg}$ para la presión sistólica $(95 \%$ de intervalo de confianza $=0,6,-2,9 \cdot \mathrm{p}=0,003) \mathrm{y}-1,5$ $\mathrm{mmHg}$ para la presión diastólica $(95 \%$ de intervalo de confianza $=0,6,-2,5, p=0,002)$. En Chile, Berríos ${ }^{46}$, realizó un estudio semejante con el proyecto MIRAME.

Las intervenciones poblacionales tienen un gran potencial para producir cambios duraderos y reducir la incidencia de la enfermedad, pero requieren de un intenso compromiso político que debe incluir aspectos tan importantes como la publicidad. Se estima que sobre los dos años de edad, los mensajes que reciben los niños tienden a cambiar sus hábitos de comida en forma brutal. Más bien que recibir información acerca de alimentos saludables, son bombardeados por una gran cantidad de avisos publicitarios, especialmente provenientes de la $\mathrm{TV}$, en relación a alimentos con altos porcentajes de grasas, elevados contenido de azúcar y especialmente de sodio. Los niños están expuestos a ver estos avisos publicitarios aproximadamente 10.000 veces por año ${ }^{47}$.

La OMS en el año 2006, en París recomienda la adquisición de políticas claras respecto al sodio en la alimentación y propone implementar tres diferentes áreas ${ }^{48}$ :

- producción de alimentos en forma industrial sin agregado de sal o con la mínima cantidad posible

- cambios en el entorno de la población, de modo de asegurar que los alimentos más sanos sean fácilmente identificables a través de etiquetado apropiado, ubicación de fácil visión de los productos alimenticios saludables en los supermercados y otros lugares de ventas y

- una activa y amplia campaña educativa en todos los grupos etarios en relación a alimentación saludable y con bajo contenido en sodio.

En la tabla 1 se muestran las recomendacio- 
Tabla 1. Ingesta diaria de sodio recomendada según edad

\begin{tabular}{lc}
\hline Edad & Ingesta de sodio por día $(\mathbf{m g})$ \\
\hline 0 a 6 meses & 120 \\
7 a 12 meses & 370 \\
1 a 3 años & 1000 \\
4 a 8 años & 1200 \\
9 o más años & 1500 \\
\hline
\end{tabular}

Tabla 2. Correlaciones en miligramos para el sodio y en gramos para la sal

\begin{tabular}{cc}
\hline Sodio en $\mathbf{~ m g}$ & Sal en $\mathbf{~}$ \\
\hline 500 & 1,25 \\
1500 & 3,75 \\
2000 & 5,0 \\
2300 & 5,8 \\
2400 & 6,0 \\
3000 & 7,5 \\
4000 & 10,0 \\
\hline
\end{tabular}

nes para una adecuada ingesta de sodio de acuerdo a la edad y en la tabla 2 la correlación entre $\mathrm{mg}$ de sodio y $\mathrm{g}$ de sal.

\section{Conclusiones}

En los últimos 20 años en Chile, se han producido descensos importantes en la tasa de mortalidad general, resultante de una indiscutible mejoría en el nivel de salud de la población (reducción de la tasa 8,7 a 5,4 por mil habitantes entre 1970 y 1995). Esta mejoría no ha sido uniforme, observándose que la reducción de enfermedades no CV es muy superior al descenso observado en las tasas por enfermedades cardiovasculares, probablemente por el desarrollo de acciones dirigidas a estimular la salud materno infantil ${ }^{49}$.

El pediatra reconoce el rol que tiene en identificar y tratar las causas curables de HA. Tradicionalmente su accionar se centra en el grupo que tiene HA secundaria a enfermedades renales, endocrinas, cardíacas o secundarias a drogas, pero debe aceptar que tiene una responsabilidad, incluso mayor, frente al gran número de niños normotensos. En ellos debe descubrir los que están en riesgo de HA, ya que es en este grupo donde la acción preventiva puede lograr cambios en la morbimortalidad en el adulto.

Frente a la evidencia que los factores de riesgos biológicos y conductuales están presentes en edades jóvenes y que éstos son más modificables en la niñez y juventud que cuando se es adulto, aparece clara la necesidad de promover cambios en estos grupos etarios. Deben intervenirse los hábitos, costumbre y tendencias de los jóvenes, escolares y adolescentes, a través de acciones con un enfoque multifactorial, familiar, grupal y comunitario. Es el pediatra quién debe asumir y liderar este desafío, en conjunto con el equipo de salud, profesores y otros organismos de la sociedad.

Hay evidencia científica del efecto adverso del excesivo consumo de sal sobre la salud, particularmente en la HA.

Las recomendaciones actuales indican que el consumo de sodio debe ajustarse a la edad del niño y en los escolares no sobrepasar los $1500 \mathrm{mg}$ diarios.

Las políticas de reducción del consumo de sal en la dieta son altamente costo-efectivas y deben implementarse acciones que estimulen la reducción del contenido de sal en los alimentos facturados, que éstos sean fácilmente reconocibles en su etiquetado y finalmente promover su consumo a través de los medios publicitarios.

\section{Referencias}

1.- Kunstman S: Epidemiología de la hipertensión arterial en Chile y Latinoamérica. En Hipertensión Saieh C, Zehnder C, ed., Santiago: Editorial Mediterráneo, 2007; 23-34.

2.- The World Health Report 2003: Shaping the future. Geneva, Switzerland: WHO 2003.

3.- Zamora L, Arellano M, Kunstmann S, et al: Invalidez en el sistema privado de pensiones. Rev Méd Chile 1997; 125: 99-106.

4.- Vasan RS, Beiser A, Seshadri S, et al: Residual lifetime risk for developing hypertension in middle-aged women and men: The Framingham Heart Study. JAMA 2002; 287: 1003-10.

5.- Beaglehole $R$, Bonita R, Jackson R, Stewart A: Prevention and control of hypertension in New Zealand: a reappraisal. N Z Med J 1988; 101: 480-3. 
6.- Rosner B, Hennekens CH, Kass EH, Miall WE: Agespecific correlation analysis of longitudinal blood pressure data. Am J Epidemiol 1977; 106: 306-13.

7.- de Swiet M, Fayers $P$, Shinebourne EA: Blood pressure in first 10 years of life: The Brompton study. BMJ 1992; 304: 23-6.

8.- Nelson MJ, Ragland DR, Syme SL: Longitudinal prediction of adult blood pressure from juvenile blood pressure levels. Am J Epidemiol 1992; 136: 633-45.

9.- Schreuder MF, Bokenkamp A, vanWijk JA: Salt intake in children: increasing concerns? (Letter). Hypertension 2007; 49: 1038.

10.- He FJ, MacGregor GA: Salt and blood pressure in children. J Hum Hypertension 2008; 22: 71-2.

11.- Norwood VF: Hypertension. Pediatr Rev 2002; 23: 197-208.

12.- Lagomarsino E, Saieh C, Aglony M: Recomendación de Ramas: Actualizaciones en el diagnóstico y tratamiento de la hipertensión arterial en pediatría. Rev Chil Pediatr 2008; 79: 62-80.

13.- Lurbe E, Torró I, Cremades B: Hipertensión arterial en niños y adolescentes. Protocolos, diagnósticos y terapéuticos. En. Nefrourología Pediátrica. Malaga S. (ed) Madrid 2005; 155-64

14.- Papademetriou V, Kokkinos PF: The role of exercise in the control of hypertension and cardiovascular risk. Curr Opin Nephrol Hypertens 1996; 5: 459-62.

15.- Beilin L, Burke V, Cox KL, Hodgson JM, Mori TA, Pudey IB: Non pharmacologic therapy and lifestyle factors in hypertension. Blood Pressure 2001; 10: 35265.

16.- Labarthe D, Ayala $C$ : Nondrug interventions in hypertension prevention and control. Cardiol Clin 2002; 20: 1-17.

17.- Svetkey L, Harsha DW, Vollmer WM, Stevens V, Obarzanek E, Elmer PJ: Premier: A clinical trial of comprehensive lifestyle modification for blood pressure control: Rationale, design and baseline characteristics. Ann Epidemiol 2003; 13: 462-71.

18.- Pickering TG: Lifestyle modification and blood pressure control. Is the glass half full or half empty? JAMA 2003; 289: 2131-2.

19.- Cavagnaro $F$, Vogel A: Problemas hidroelectrolíticos y del equilibrio ácido-básico. En Manual de Neonatología Tapia JL, Ventura Juncá P. ed. Santiago. Ed. Mediterráneo 2000; 479-97.

20.- Gayton AC: The kidney in blood pressure control and hypertension. En Pediatric Nephrology. Holliday MA, Barrat TM, Venier RL, ed. Baltimore. Ed Williams \& Wilkins, 1987; 729-37.

21.- Cruz Coke R: Los genes de la hipertensión arterial humana. Rev Med Chile 1997; 125: 351-7.

22.- Barker DJP, Bull AR, Osmond C, Simmonds SJ: Fetal and placental size and risk of hypertension in adult life. BMJ 1990; 301: 259-62.

23.- Law CM, Sweet $M$, Osmond C, Fayers PM, Barker DJP: Initiation of hypertension in uterus and its amplification throughout life. BMJ 1993; 306: 247.

24.- Barker DJP, Fall CDH: Fetal and infant origins of cardiovascular diseases. Arch Dis Child 1993; 68: $797-$ 9.

25.- AAP: Committee on Nutrition-Commentary on breastfeeding and infant formulas including standards for formulas. Pediatrics 1976; 57: 278-85.

26.- Ingelfinger J: Hypertension. En Pediatric Kidney Disease. Edelman Ch. ed. Boston, Toronto, London. ED. Little, Brown and Co. 1992: 581-92.

27.- Pérez-Olea J: Factores en la etiopatogenia de la hipertensión arterial. Rev Méd Chile 1992; 120: 6749.

28.- Fouad-Tarazi FM: Hypertension hemodynamics. Med Clin North Am 1997; 81: 1131-45.

29.- Eaton SB, Konner M: Paleolithic nutrition. N Engl J Med 1985; 312: 283-9.

30.- de Wardener HE, MacGregor GA: Sodium and blood pressure. Curr Opin Cardiol 2002, 17: 360-7.

31.- Denton D, Weisinger R, Mundy NI, et al: The effect of increased salt intake on blood pressure of chimpanzees. Nat Med 1995; 1: 1009-16.

32.- Eichberg JW, Shade RE: Normal blood pressure in chimpanzees. J Med Primatol 1987; 16: 317-21.

33.- Elliot P, Stamler J, Nichols R: Intersalt revisited: Further analyses of 24 hours sodium excretion and blood pressure within and across populations. BMJ 1996; 312: 1249-53.

34.- Cruz-Coke R, Etcheverry R, Nagel RL: The influence of migration on blood pressure of Easter islanders. Lancet 1964; 1: 697-701.

35.- Karppanen H, Mervaala E: Sodium intake and hypertension. Prog Cardiovasc Dis 2006; 49: 59-75.

36.- He FJ, MacGregor GA: Importance of salt in determining blood pressure in children: metaanalysis of controlled trials. Hypertension 2006; 48: 861-9.

37.- Bacon SL, Sherwood A, Hinderliter A, Blumenthal $J A$ : Effects of exercise, diet and weight loss on high blood pressure. Sport Med 2004; 34: 307-16.

38.- Rouse IL, Beilin LJ, Armstrong BK, Vandongen R: Blood-pressure-lowering effect of a vegetarian diet: controlled trial in normotensive subjects. Lancet 1983; 1: 5-10.

39.- Saieh $C$, Zehnder $C$ : Estilos de vida saludables e hipertensión arterial. En Hipertensión. Saieh C, Zehnder C. ed. Santiago, Chile. Ed. Mediterráneo, 2007; 483-90.

40.- Mattes RD, Donnelly D: Relative contributions of dietary sodium sources. J Am Coll Nutr 1991; 10: 383-93.

41.- Milton C, Estrada C: Determinación de cloruro de sodio en las raciones alimenticias del programa de alimentación escolar de la JUNAEB. Tesis presentada para optar al grado de Licenciado en Ciencias de los Alimentos. Escuela de Ingeniería de los Alimentos. Universidad Austral. Valdivia 2007.

42.- Chobanian AV, Hill M: National Heart Lung and Blood Institute Workshop on sodium and blood pressure: a critical review of current scientific evidence. Hypertension 2000; 35: 858-63.

43.- Lawes CMM, Vander Hoorn S, Law MR, Elliott P, 
MacMahon S, Rodgers A: Blood pressure and the global burden of disease 2000. Part II: Estimates of attributable burden. J Hypertens 2006; 24: 423-30.

44.- Tekol $Y$ : Irreversible and reversible components in the genesis of hypertension by sodium chloride (salt). Med Hypotheses 2008; 70: 255-9.

45.- Tekol Y: Salt addiction: A different kind of drug addiction. Med Hypotheses 2006; 67: 1233-4.

46.- Lagomarsino E: Rol del pediatra en la prevención de la hipertensión arterial esencial del adulto. Rev Méd Chile 1999; 127: 349-57.

47.- Horgen KB, Choate M, Brownell KD: En: Handbook of Children and the Media: Singer DG, Singer JL (eds). CA. Ed. Thousand Oaks, 2002: 78.

48.- Report of a WHO Forum and Technical meeting. October 2006, Paris, France.

49.- Berrios $X$ : Tendencia temporal de los factores de riesgo de enfermedades crónicas. ¿La antesala silenciosa de una epidemia que viene? Rev Méd Chile 1997; 12: 1405-7.

50.- Scientific advisory committee on nutrition (SACN) and Great Britain Department of Health Food Standard Agency (DH): Salt and health. The Stationary Office. London, U.K. 2003; 134. 